\title{
Extrapyramidal side effects after metoclopramide administration in a post-anesthesia care unit
} -A case report-

\author{
Youn Yi Jo, Yong Beom Kim, Mi Ran Yang, and Young Jin Chang \\ Department of Anesthesiology and Pain Medicine, Gachon University of Medicine and Science Gil Medical Center, Incheon, Korea
}

Although the incidence of extrapyramidal reactions associated with metoclopramide has been reported to be approximately $0.2 \%$, such reactions are rare in the anesthetic field. Several anesthetic adjuvants, including ondansetron and pregabalin, have also been associated with extrapyramidal side effect. Here, the authors report the case of a 47-year-old patient, previously administered pregabalin and ondansetron, who developed extrapyramidal side effects after a single injection of metoclopramide $(10 \mathrm{mg})$ in a post-anesthesia care unit. (Korean J Anesthesiol 2012; 63: 274-276)

Key Words: Extrapyramidal side effect, Metoclopramide, Post-anesthesia care unit.

Metoclopramide has been used as an anesthetic adjunct due to its prokinetic and antiemetic effects, and conventionally, metoclopramide, at an antiemetic dose of $10 \mathrm{mg}$, has been used to prevent postoperative nausea and vomiting (PONV), because at this dosage it is not believed to have adverse effects, such as, extrapyramidal symptoms [1]. Although the incidence of extrapyramidal reactions associated with metoclopramide have been reported to be approximately $0.2 \%$ [2], such symptoms are only rarely encountered in the anesthetic field.

No previous report has been issued of extrapyramidal side effects, including an oculogyric crisis, after metoclopramide in a post-anesthetic care setting in the English literature. Here, we report a case of extrapyramidal side effect development after a single injection metoclopramide $(10 \mathrm{mg})$ in a post-anesthesia care unit in a patient, previously administered pregabalin and ondansetron.

\section{Case Report}

A 47-year-old (height, $166 \mathrm{~cm}$; weight, $65 \mathrm{~kg}$ ) man presented at our emergency room with low back pain. His medical history was unremarkable, and a magnetic resonance image (MRI) scan demonstrated spondylolytic spondylolisthesis and disc protrusion at the L3-S1 level. For pain control, he had taken 3 tablets/day of tramadol hydrochloride/acetaminophen (ultracet ${ }^{\circledR}$ ), $200 \mathrm{mg} /$ day of aceclofenac $\left(\right.$ ASEC $^{\circledR}$ ), and $150 \mathrm{mg} /$ day of pregablin $\left(\right.$ lyrica $^{\circledR}$ ) for 5 days before operation. All vital signs and laboratory data before the surgery were within normal limits.

Received: July 11, 2011. Revised: 1st, August 19, 2011; 2nd, September 19, 2011; 3rd, October 14, 2011. Accepted: October 14, 2011.

Corresponding author: Young Jin Chang, M.D., Department of Anesthesiology and Pain Medicine, Gachon University of Medicine and Science Gil Medical Center, 1198, Guwol-dong, Namdong-gu, Incheon 405-760, Korea. Tel: 82-32-460-3637, Fax: 82-32-469-6319, E-mail: yjsk@gilhospital.com (c) This is an open-access article distributed under the terms of the Creative Commons Attribution Non-Commercial License (http:// creativecommons.org/licenses/by-nc/3.0/), which permits unrestricted non-commercial use, distribution, and reproduction in any medium, provided the original work is properly cited. 
Intramuscular midazolam $2 \mathrm{mg}$ and glycopyrrolate 0.2 mg were administered as premedication 30 minutes before anesthesia, and upon arrival at the operating room, standard monitoring devices were applied. Before anesthetic induction his vital signs were; blood pressure 144/81 mmHg, heart rate 62 beats/min, and $97 \%$ saturation by pulse oximetry. After preoxygenation, anesthesia was induced with $120 \mathrm{mg}$ of propofol, $100 \mathrm{ug}$ of fentanyl, and $50 \mathrm{mg}$ of rocuronium. Endotracheal intubation was performed using a wire reinforcement endotracheal tube with a single lumen of internal diameter (ID) 7.5 $\mathrm{mm}$ in one attempt without difficulty. A radial arterial line and central venous catheter in the right internal jugular vein as well as a Foley catheter were inserted. Anesthesia was maintained with desflurane in $\mathrm{N}_{2} \mathrm{O} / \mathrm{O}_{2}\left(\mathrm{FiO}_{2}=0.5\right)$ with supplemental continuous remifentanil infusion. Lumbar discectomy with posterior fusion proceeded uneventfully. The patient received fentanyl (1,000 ug) mixed with $100 \mathrm{ml}$ of normal saline for 48 $\mathrm{h}$ using patient-controlled intravenous analgesia (PCA). To prevent postoperative nausea and vomiting, ondansetron 8 mg was given intravenously before the end of surgery, when all anesthetic agents were discontinued and the residual neuromuscular blockade was reversed with pyridostigmine and glycopyrrolate. The tracheal tube was removed when the patient responded to verbal commands and showed sufficient spontaneous respiration and neuromuscular function. He was the transferred to the post-anesthesia care unit (PACU) where his vital signs were; 145/85 $\mathrm{mmHg}, 88$ beats/min, and saturation $97 \%$. Five liters of oxygen was applied via a facial mask and a neurosurgeon checked motor and sensory functions. A few minutes later, he complained of nausea and $10 \mathrm{mg}$ of metoclopramide was administered intravenously. Approximately 10 minutes after this administration, he complained that he could not see his nose. On examination, it was found that he had a bilaterally fixed upward, lateral gaze. His pupils were equally round, reactive to light bilaterally, and he had no photophobia or blurred vision. At the time, he was in a rigid, opisthotonic posture with his neck fixed backward and laterally. His consciousness was clear, and he was able to answer all questions; vital signs were 162/90 $\mathrm{mmHg}$ and 98 beats/min. Intravenous midazolam $2 \mathrm{mg}$ was administered once, and about 5 minutes later the symptom began to abate and about 20 minutes later the dystonic reaction had completely resolved. Arterial blood gas analysis and other laboratory findings were within normal limits. The patient remained in the PACU for 2 hours uneventfully, and was then transferred to the general ward. He did not experience any further dystonic reaction during the remainder of his hospitalization and was discharged on postoperative day 8. Seven days after discharge, he experienced abdominal pain and was re-admitted for laparoscopic cholecystectomy. He was administered ondansetron $8 \mathrm{mg}$ intravenously before end of surgery, no dystonic reaction occurred in the PACU, and he was discharged on postoperative day 3 .

\section{Discussion}

Here, we present the case of a male patient who experienced an extrapyramidal side effect following a single administration of metoclopramide for PONV following spine surgery. A few cases of oculogyric crisis have been described after metoclopramide, but most involved pediatric patients or adult patients receiving metoclopramide regularly [2,3].

Metoclopramide (a chlorobenzamide) is a useful anesthetic adjuvant due to its antiemetic and prokinetics effects. Its antiemetic effect is the result of dopamine D2 receptor antagonism in the chemoreceptor trigger zone in the central nervous system. Extrapyramidal reactions are the most common acute side effect of metoclopramide with a reported incidence of $0.2 \%$, but in the aged and young patients this incidence increase up to as high as $25 \%$ [4]. Even though the mechanism underlying extrapyramidal reactions is unclear, a striatal dopamine D2 receptor blockade is believed to be the principal cause [5]. Extrapyramidal symptoms may be encountered at the recommended dose and usually occur within the first 24-72 hour of administration [6]. However, our patient experienced an extrapyramidal side effect only 10 minutes after metoclopramide was injected, and is consistent with a previous report of extrapyramidal symptom development within 10-15 minutes of intravenous metoclopramide (10 mg) administered to prevent pulmonary aspiration prophylaxis [7].

Acute extrapyramidal symptoms tend to resolve rapidly and without serious sequela after the cessation or reduction of the causative drugs. Intravenous anticholinergic benztropine is effective for most dystonic reactions within 5 minutes. Antihi stamine, benzodiazepines, beta-adrenergic antagonists (propranolol), beta-adrenergic agonists (clonidine), or dopamine agonists (amantadine) may also be used [8]. In the described case, we used midazolam ( $2 \mathrm{mg}$, iv.) because it is readily accessible in the anesthetic field and our patient recovered rapidly.

Despite the close temporal relationship between extrapyramidal symptom development and metoclopramide administration, we cannot exclude the possibility of cross-reactivity with other anesthetic adjuvant. In particular, in view of the time of onset of the side effect, ondansetron should also be considered as a causative factor even though no dystonic reaction was elicited by ondansetron during the second operation. The oculogyric crisis in our patient occurred approximately 10 minutes after using metoclopramide and within 30 minutes after using ondansetron, and the onset of ondansetron-induced 
extrapyramidal reaction reportedly varies from several minutes to 30 minutes $[9,10]$.

Ondansetron is a selective 5-HT3 receptor antagonist and is considered free of neurologic complication, including extrapyramidal reactions, because it is not a central dopaminergic blocker. However, although ondansetron does not bind to dopamine receptors, it does play an important role in dopaminergic transmission by reducing mesolimbic dopamine activity [11]. Our patient also used pregabalin before surgery, and pregabalin binds with high affinity to voltage-gated calcium channels in central nervous system and modulates the calciumdependent releases of several neurotransmitters [12]. In one case report only, dystonia occurred when pregabalin was discontinued and subsided when pregabalin was re-introduced only to reappear again when pregabalin was again discontinued [13]. Our patient took pregabalin daily before surgery and no dystonic reactions occurred after it was discontinued postoperatively.

In the described case, we reintroduced ondansetron to prevent PONV after laparoscopic cholecystectomy, despite his extrapyramidal side effect history, because he also had a history of PONV during previous surgery. Furthermore, he underwent laparoscopic surgery with fentanyl based PCA and did not take other drugs that could influence dopaminergic receptor after spine surgery. Although we could not exclude the possibility that ondansetron was the cause of previous dystonic reaction, in view of the known incidences of extrapyramidal side effects, we choose ondansetron rather than metoclopramide.

Commonly used anti-emetics like metoclopramide and ondansetron can induce extrapyramidal reactions even after a single injection. Anesthesiologists should have a high index of suspicion about anti-emetics induced extrapyramidal side effect and must decide on correct indications before prescribing anti-emetics with a known extrapyramidal side effect.

\section{References}

1. Watcha MF, White PF. Postoperative nausea and vomiting. Its etiology, treatment, and prevention. Anesthesiology 1992; 77: 16284.

2. Yis U, Ozdemir D, Duman M, Unal N. Metoclopramide induced dystonia in children: two case reports. Eur J Emerg Med 2005; 12: 117-9.

3. Lou E, Abou-Zeid N. A case of metoclopramide-induced oculogyric crisis in a 16-year-old girl with cystic fibrosis. South Med J 2006; 99 : 1290-1.

4. Montvale NJ. Metoclopramide. In: Physicians' desk reference. 50th ed. Montvale, New Jersey, Medical Economics. 1996, pp 2068-70.

5. Kapur S, Zipursky R, Jones C, Remington G, Houle S. Relationship between dopamine $\mathrm{D}(2)$ occupancy, clinical response, and side effects: a double-blind PET study of first-episode schizophrenia. Am J Psychiatry 2000; 157: 514-20.

6. Tait PA. Supraglottic dystonic reaction to metoclopramide in a child. Med J Aust 2001;174: 607-8.

7. Moos DD, Hansen DJ. Metoclopramide and extrapyramidal symptoms: a case report. J Perianesth Nurs 2008; 23: 292-9.

8. Kamin J, Manwani S, Hughes D. Emergency psychiatry: extrapyramidal side effects in the psychiatric emergency service. Psychiatr Serv 2000; 51: 287-9.

9. Sprung J, Choudhry FM, Hall BA. Extrapyramidal reactions to ondansetron: cross-reactivity between ondansetron and prochlorperazine? Anesth Analg 2003; 96: 1374-6.

10. Spiegel JE, Kang V, Kunze L, Hess P. Ondansetron-induced extrapyramidal symptoms during cesarean section. Int J Obstet Anesth 2005; 14: 368-9.

11. Wilde MI, Markham A. Ondansetron. A review of its pharmacology and preliminary clinical findings in novel applications. Drugs 1996; 52: 773-94.

12. Kim JC, Choi YS, Kim KN, Shim JK, Lee JY, Kwak YL. Effective dose of peri-operative oral pregabalin as an adjunct to multimodal analgesic regimen in lumbar spinal fusion surgery. Spine (Phila Pa 1976) 2011; 36: 428-33.

13. Karosin C, Kofler M, Mayr A, Saltuari L. Pregabalin: a treatment option for dystonia? Neurol Sci 2012; 33: 351-4. 